\title{
Bone turnover markers
}

Sunethra Devika C Thomas

Chemical pathologist/fellow SA Pathology

Division of Chemical

Pathology

Adelaide

Key words

alkaline phosphatase, procollagen peptides, pyridinoline crosslinks, telopeptides

\section{SUMMARY}

Markers of bone turnover are proteins originating from osteoclast and osteoblast activity or fragments released during the formation or degradation of type I collagen.

Some of these peptides are sufficiently small to be filtered into urine, while larger fragments may be detected in blood.

These markers may provide an assessment of the rate of bone turnover, however they are affected by a variety of physiological and pathological factors.

They cannot be used for screening or the diagnosis of specific diseases.

\section{Introduction}

Bone is a dynamic and living tissue and bone remodelling occurs throughout life. The remodelling cycle consists of resorption and formation. Products of the bone remodelling processes are termed bone turnover markers. There are bone formation markers and bone resorption markers. These markers are a quantitative and dynamic reflection of current bone turnover, whereas bone density measurements reflect events that affected bone turnover over the preceding months to years.

\section{Bone remodelling cycle}

The bone remodelling cycle begins with the recruitment of osteoclast precursor cells. These differentiate into osteoclasts when they receive signals from osteoblasts. Mature osteoclasts then synthesise and release proteolytic enzymes that digest the collagen matrix. This bone resorption is the first phase of the remodelling cycle. The length of this phase is regulated by apoptosis of osteoclasts. In the next phase of the remodelling cycle preosteoblasts are attracted from mesenchymal stem cells in the bone marrow. Mature osteoblasts synthesise the bone matrix, mainly type I collagen, and regulate the mineralisation of the newly formed bone. Some mature osteoblasts may be trapped within mineralised bone and become osteocytes.

\section{Bone formation markers}

Bone formation markers are products of osteoblasts and their activity.

\section{Propeptides of type I procollagen}

Type I collagen is part of the bone matrix. Osteoblasts release its precursor, type I procollagen. This undergoes proteolytic cleavage resulting in aminoterminal and carboxy-terminal propeptides of type I collagen (PINP, PICP). The concentrations of PINP and PICP in the circulation are thought to reflect the rate of bone formation. Data from a multicentre trial of teriparatide (parathyroid hormone) versus placebo suggested a relationship between an early rise in PINP and later changes in lumbar spine bone density in the teriparatide treated group.

\section{Alkaline phosphatase}

The total serum alkaline phosphatase consists of several isoforms. These isoforms originate from liver, bone, intestine, spleen, kidney and placenta. In healthy adults, about $50 \%$ of the serum alkaline phosphatase is considered to be of hepatic origin and the rest is of bone origin. Bone-specific alkaline phosphatase is synthesised in osteoblasts and reflects osteoblast activity during bone formation.

Various physical and chemical methods are used to differentiate liver and bone isoforms in serum. In the absence of liver disease and with other liver enzymes within normal limits, a raised total alkaline phosphatase is considered to represent a rise in bonespecific alkaline phosphatase. Bone-specific alkaline phosphatase is not routinely measured due to the cost.

The concentration of bone-specific alkaline phosphatase is significantly associated with fracture risk regardless of bone mineral density in postmenopausal women. ${ }^{2}$ Bone-specific alkaline phosphatase can be used to monitor progress in Paget's disease, although total alkaline phosphatase represents a cheaper and equally valid measure.

\section{Osteocalcin}

Osteocalcin is a protein synthesised by osteoblasts which binds to hydroxyapatite in the bone matrix. In addition to its function in regulating bone remodelling via a negative feedback mechanism, it is also an endocrine factor regulating glucose homeostasis. Osteocalcin is unstable once collected therefore testing is not widely offered. While low osteocalcin has been associated with an increased risk of fractures, no significant relationship was seen in prospective trials. 


\section{Bone resorption markers}

Markers of resorption are type I collagen degradation products. They reflect the rate of bone matrix breakdown and, indirectly, the number of active osteoclasts.

Hydroxyproline is an amino acid found in type I collagen of bone. Urinary excretion rate of hydroxyproline was used in the past to assess bone resorption rate. This assay has been superseded by more specific assays.

\section{Pyridinoline crosslinks}

Pyridinoline and deoxypyridinoline are small, cyclic amino structures linking peptide chains of collagen molecules. During resorption these structures are released into the circulation. These small molecules can be detected in urine, where about $40 \%$ are bound to various proteins. The urinary concentration of pyridinoline and deoxypyridinoline reflect the rate of collagen degradation.

The fraction bound to protein is not clinically significant, however it is a consideration when various methods of measurement are compared. The concentrations are not generally affected by diet, but are subject to diurnal variation. An early morning sample or a 24-hour urine collection is recommended.

\section{Telopeptides}

The $\mathrm{N}$ - and C-terminal ends of mature collagen are released during bone resorption and can be detected in the circulation. Although $\mathrm{N}$-terminal telopeptides can be measured in serum, serum concentrations of the C-terminal telopeptide of mature collagen are more useful in monitoring progress in osteoporosis and in bone resorption in multiple myeloma. A raised concentration has been associated with an increased risk of fractures independent of bone mineral density. Measurement may also be useful in monitoring the response to antiresorptive drugs such as bisphosphonates.

Tests for C-terminal telopeptide show high variability within individuals and between individuals. They are affected by marked diurnal variation and food. In addition, concentrations rise with the menopause. It is difficult to determine a reference interval so desirable limits are proposed instead. An early morning fasting blood sample is recommended.

\section{Factors influencing test results}

Bone turnover markers are released during normal bone turnover. The concentrations may rise in metabolic bone diseases (for example osteoporosis), other pathological conditions and during physiological processes such as fracture healing and growth spurts. Bone turnover markers are not disease specific.
They cannot be used for screening or diagnosis of specific bone diseases. Their concentrations and patterns may be used by specialist units to monitor treatment response and disease progression in several metabolic bone diseases including postmenopausal osteoporosis, corticosteroid-induced osteoporosis and Paget's disease.

Several factors influence the concentration of bone turnover markers in blood or urine including age, sex, fasting or non-fasting, circadian rhythms, menstrual cycle, exercise history and medical history. The interpretation of results is optimised by taking a careful clinical history and collecting specimens under standard conditions.

Age exerts the greatest effect on bone turnover markers. Concentrations are higher in children and adolescents than in adults. There may be significant increases in markers during growth spurts. In females, bone turnover markers reach a plateau between 20 and 25 years of age, and in males between 25 and 30 years of age reflecting peak bone mass. After the menopause bone turnover increases markedly, as a result of falling oestrogen, and then gradually declines but does not return to premenopausal levels. In contrast, bone turnover decreases in men with ageing

The intake of food influences bone turnover. Dietary calcium appears to inhibit bone resorption. Calcium supplements taken in the evening significantly reduce resorption markers, in the fasting state, the next morning.

Bone turnover markers have a diurnal rhythm, peaking in the morning. Seasonal variations have been reported. Exercise affects bone turnover markers and immobility results in a marked increase in bone resorption.

\section{Bone turnover markers in clinical practice}

Current evidence suggests that bone turnover markers may be useful in some patients with conditions such as osteoporosis, 3,4 for monitoring the response to antiresorptive therapy. ${ }^{5,6}$ Intravenous and oral bisphosphonate therapy respectively lead to a decrease in bone resorption markers within days and weeks. The decrease in resorption markers is followed by a decline in bone formation markers. The decrease in bone turnover markers may be sustained for years after cessation of therapy in patients who have been treated for several years.

A significant change in bone turnover markers after starting therapy confirms compliance. A decline 


\section{SELF-TEST} QUESTIONS

True or false?

7. Alkaline phosphatase is not a specific marker for bone turnover.

8. Bone turnover markers can be used to screen for bone diseases.

Answers on page 171 of up to $65 \%$ of the baseline bone turnover markers (particularly C-terminal telopeptide) may be expected after potent antiresorptive therapy such as bisphosphonates or denosumab. However, after treatment with oestradiol, the oestrogen analogue raloxifene or strontium ranelate, the fall in C-terminal telopeptide may be less. The magnitude of suppression of baseline bone turnover markers reflects the magnitude of suppression of bone turnover. With oral bisphosphonate treatment, it is not uncommon for the C-terminal telopeptide to fall below 100 nanogram/L. These changes in bone turnover particularly affect bone resorption and have been associated with a rise in bone density.

\section{Osteonecrosis of the jaw}

Osteonecrosis of the jaw has been associated with potent antiresorptive therapy such as bisphosphonates and denosumab. The risk following dental extraction may be higher in immunocompromised patients, those with bone metastases and in patients receiving chemotherapy. The risk of osteonecrosis of the jaw should be determined based on the clinical and drug history. Identification of oversuppression of bone turnover and of those susceptible to osteonecrosis of the jaw may be possible with further experience in bone resorption markers. Currently however, there is insufficient evidence to routinely use bone markers to predict the risk of developing osteonecrosis of the jaw.

\section{Conclusion}

Two markers currently used in clinical practice are serum C-terminal telopeptide and aminoterminal propeptide of type I collagen. These markers are dynamic and reflect the resorption and formation rates. However, other analytes such as alkaline phosphatase, parathyroid hormone and 25-hydroxyvitamin D should also be considered. While these markers reflect the rate of bone turnover, they currently have limited clinical utility. They do not predict fracture risk and they are not validated as screening tests in routine clinical practice. Various fracture risk calculators currently available do not include bone turnover markers due to the lack of standardisation of analytical methods and the lack of common reference intervals. $<$

Conflict of interest: none declared

\section{REFERENCES}

1. Tsujimoto M, Chen P, Miyauchi A, Sowa H, Krege JH. PINP as an aid for monitoring patients treated with teriparatide. Bone 2011;48:798-803.

2. Garnero P, Sornay-Rendu E, Claustrat B, Delmas PD. Biochemical markers of bone turnover, endogenous hormones and the risk of fractures in postmenopausal women: the OFELY study. J Bone Miner Res 2000;15:1526-36.

3. Rosen HN. Use of biochemical markers of bone turnover in osteoporosis. UpToDate, Inc. 2012. www.uptodate.com/contents/use-of-biochemical-markersof-bone-turnover-in-osteoporosis [cited 2012 Sep 3]

\section{FURTHER READING}

Marx RE, Cillo JE, Ulloa JJ. Oral bisphosphonate-induced osteonecrosis: risk factors, prediction of risk using serum CTX resting, prevention and treatment. J Oral Maxillofac Surg 2007;65:2397-410

Osteoporosis Australia, Australian Dental Association. Bisphosphonates and ONJ (osteonecrosis of the jaw). 2010 www.osteoporosis.org.au/images/stories/documents/internal/ Joint\%200A\%20and\%20ADA\%200NJ\%20Statement.pdf [cited 2012 Sep 3]
4. Talwar SA. Bone markers in osteoporosis. New York: WebMD Health Professional Network.

http://emedicine.medscape.com/article/128567-overview [cited 2012 Sep 3]

5. Compson J. Monitoring bone mineral density during antiresorptive treatment for osteoporosis [editorial]. BMJ 2009;338:b1276.

6. Bonnick SL, Shulman L. Monitoring osteoporosis therapy: bone mineral density, bone turnover markers or both? Am J Med 2006;119 Suppl 1:S25-31. management of patients receiving oral bisphosphonate therapy: expert panel recommen J Am Dent Assoc 2006;137:1144-50.

Vasikaran S, Eastell R, Bruyère O, Foldes AJ, Garnero P, Griesmacher A, et al; IOF-IFCC Bone Marker Standards Working Group. Markers of bone turnover for the prediction of fracture risk and monitoring of osteoporosis treatment: a need for international reference standards. Osteoporos Int 2011;22:391-420. 